\title{
Tunneling magnetoresistance phenomenon utilizing graphene magnet electrode
}

\author{
Hashimoto, T.; Kamikawa, S.; Soriano, D.; Pedersen, Jesper Goor; Roche, S.; Haruyama, J.
}

Published in:

Applied Physics Letters

Link to article, DOI:

$10.1063 / 1.4901279$

Publication date:

2014

Document Version

Publisher's PDF, also known as Version of record

Link back to DTU Orbit

Citation (APA):

Hashimoto, T., Kamikawa, S., Soriano, D., Pedersen, J. G., Roche, S., \& Haruyama, J. (2014). Tunneling magnetoresistance phenomenon utilizing graphene magnet electrode. Applied Physics Letters, 105(18), [183111]. https://doi.org/10.1063/1.4901279

\section{General rights}

Copyright and moral rights for the publications made accessible in the public portal are retained by the authors and/or other copyright owners and it is a condition of accessing publications that users recognise and abide by the legal requirements associated with these rights.

- Users may download and print one copy of any publication from the public portal for the purpose of private study or research.

- You may not further distribute the material or use it for any profit-making activity or commercial gain

- You may freely distribute the URL identifying the publication in the public portal 


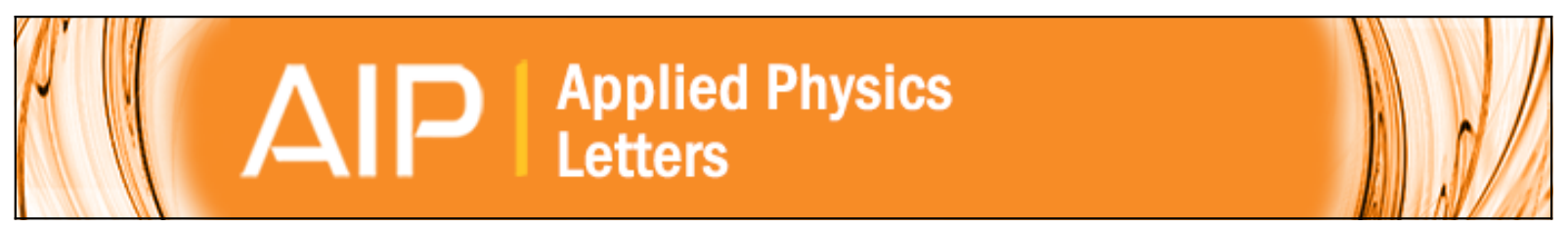

\section{Tunneling magnetoresistance phenomenon utilizing graphene magnet electrode}

T. Hashimoto, S. Kamikawa, D. Soriano, J. G. Pedersen, S. Roche, and J. Haruyama

Citation: Applied Physics Letters 105, 183111 (2014); doi: 10.1063/1.4901279

View online: http://dx.doi.org/10.1063/1.4901279

View Table of Contents: http://scitation.aip.org/content/aip/journal/apl/105/18?ver=pdfcov

Published by the AIP Publishing

\section{Articles you may be interested in}

Spin transport and magnetoresistance in Thue-Morse graphene superlattice with two ferromagnetic graphene electrodes

J. Appl. Phys. 114, 163715 (2013); 10.1063/1.4827380

Effect of electrode composition on the tunnel magnetoresistance of pseudo-spin-valve magnetic tunnel junction with a MgO tunnel barrier

Appl. Phys. Lett. 90, 212507 (2007); 10.1063/1.2742576

Magnetoresistance behavior of a magnetic tunnel junction with perpendicularly magnetized $\mathrm{Co} / \mathrm{Pd}$ multilayers J. Appl. Phys. 97, 10C902 (2005); 10.1063/1.1845932

Spin-transfer effects in nanoscale magnetic tunnel junctions

Appl. Phys. Lett. 85, 1205 (2004); 10.1063/1.1781769

Magnetic tunnel junctions with high magnetoresistance and small bias voltage dependence using epitaxial $\mathrm{NiFe}(111)$ ferromagnetic bottom electrodes

J. Appl. Phys. 93, 8555 (2003); 10.1063/1.1544458

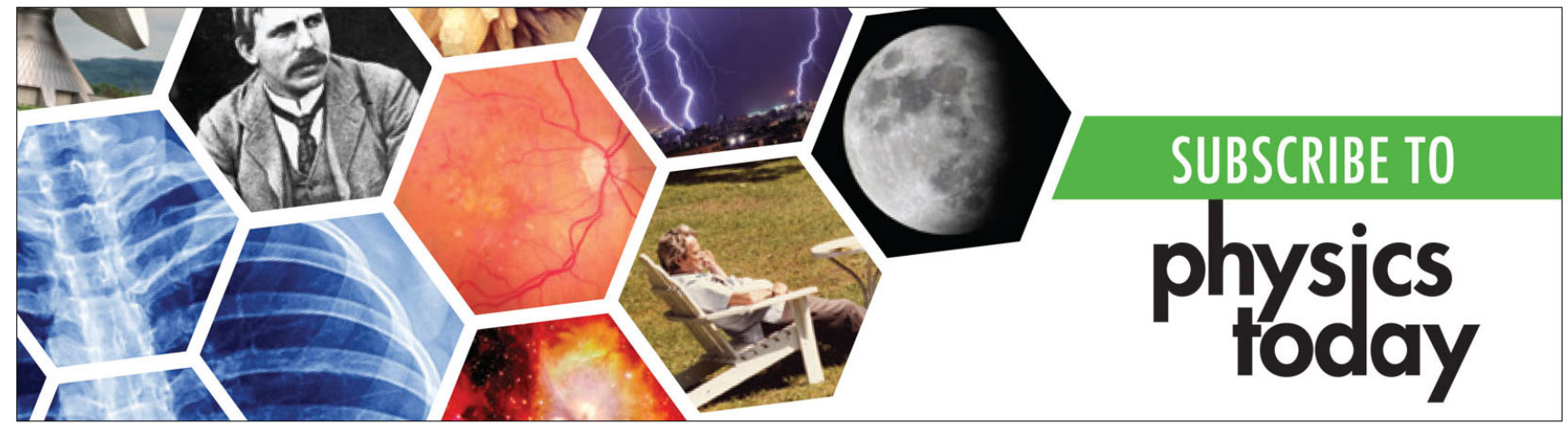




\title{
Tunneling magnetoresistance phenomenon utilizing graphene magnet electrode
}

\author{
T. Hashimoto, ${ }^{1}$ S. Kamikawa, ${ }^{1}$ D. Soriano, ${ }^{2}$ J. G. Pedersen,${ }^{2,3}$ S. Roche ${ }^{2,4}$ \\ and J. Haruyama ${ }^{1, a)}$ \\ ${ }^{1}$ Faculty of Science and Engineering, Aoyama Gakuin University, 5-10-1 Fuchinobe, Sagamihara, \\ Kanagawa 252-5258, Japan \\ ${ }^{2}$ Institut Català de Nanociència i Nanotecnologia (ICN2), Campus de la UAB, Edifici ICN2, \\ 08193 Bellaterra, Barcelona, Spain \\ ${ }^{3}$ Department of Micro-and Nanotechnology, DTU Nanotech, Technical University of Denmark, \\ DK-2800 Kongens Lyngby, Denmark \\ ${ }^{4}$ ICREA - Institucio Catalana de Recerca i Estudis Avancats, 08010 Barcelona, Spain
}

(Received 4 September 2014; accepted 28 October 2014; published online 7 November 2014)

\begin{abstract}
Using magnetic rare-metals for spintronic devices is facing serious problems for the environmental contamination and the limited material-resource. In contrast, by fabricating ferromagnetic graphene nanopore arrays (FGNPAs) consisting of honeycomb-like array of hexagonal nanopores with hydrogen-terminated zigzag-type atomic structure edges, we reported observation of polarized electron spins spontaneously driven from the pore edge states, resulting in rare-metal-free flatenergy-band ferromagnetism. Here, we demonstrate observation of tunneling magnetoresistance (TMR) behaviors on the junction of cobalt/ $/ \mathrm{SiO}_{2} / \mathrm{FGNPA}$ electrode, serving as a prototype structure for future rare-metal free TMR devices using magnetic graphene electrodes. Gradual change in TMR ratios is observed across zero-magnetic field, arising from specified alignment between poreedge- and cobalt-spins. The TMR ratios can be controlled by applying back-gate voltage and by modulating interpore distance. Annealing the $\mathrm{SiO}_{2} / \mathrm{FGNPA}$ junction also drastically enhances TMR ratios up to $100 \%$. (C) 2014 AIP Publishing LLC. [http://dx.doi.org/10.1063/1.4901279]
\end{abstract}

Several key components of spintronics have been realized in recent years, e.g., giant magnetoresistance (GMR), tunneling MR (TMR), and spin valve devices. ${ }^{1-3}$ Giant TMR ratios, $\left(R_{\mathrm{AP}}-R_{\mathrm{P}}\right) / R_{\mathrm{P}}$, where $\mathrm{AP}$ and $\mathrm{P}$ refer to antiparallel and parallel orientations of the spin configurations of the two electrodes, of $\sim 1000 \%$ have been obtained in $\mathrm{CoFeB} / \mathrm{MgO} /$ $\mathrm{CoFeB}$ junctions. A wide variety of materials have been utilized for spintronic devices such as ferromagnetic metals and $^{1-3}$ ferromagnetic semiconductors. ${ }^{4-6}$ In all cases, however, rare magnetic elements are essential to provide polarized spins to the systems.

In contrast, it was theoretically predicted that graphene edges with specified atomic structures (the so-called zigzag edge, Fig. 1(b)) are spontaneously spin polarized, exhibiting flat-energy-band ferromagnetism caused from extremely high electronic density of spin states (i.e., the edge states) and a strong spin interaction among the localized electrons. ${ }^{7-9}$ Importantly, this occurs despite the absence of rare magnetic elements, considering just carbon atoms with $s p^{2}$ orbitals. In the case of graphene nanoribbons (GNR, Fig. 1(b)), ${ }^{7,10}$ the appearance of the spin polarization is highly sensitive to the spin interaction between the two edges and is determined so as to maximize exchange energy gain (similar to Hund's rule in atoms). In particular, spin ground states in GNRs under absent magnetic fields are still under debating stages. These properties suggest that novel types of spin-based devices may be realized using graphene-based materials, without the need

\footnotetext{
${ }^{\text {a) }}$ Author to whom correspondence should be addressed. Electronic mail: J-haru@ee.aoyama.ac.jp
}

of rare magnetic atoms. Long spin diffusion lengths and the introduction of the spin-orbit interaction by hydrogenation ${ }^{11,12}$ are further recent examples of the potential of graphene spintronics.

In prior works, we have experimentally confirmed the formation of this flat-band ferromagnetism in hydrogen $(\mathrm{H})$ terminated zigzag-edged GNPAs (Figs. 1(a) and 1(b)). ${ }^{13-16}$ Because the GNPA corresponds to a large ensemble of the zigzag GNRs (i.e., interpore regions), it is effective to detect small magnetic and electric signals arising from the pore edge spins. Observation of the significant reduction of the G/D peak ratios by the critical-temperature annealing $\left(\sim 800^{\circ} \mathrm{C}\right)$ in Raman spectrum and the comparison with the previous other experiments (e.g., extremely low G/D peak ratios in the intentionally fabricated zigzag-edge hexagonal pores ${ }^{17}$ and in the zigzag-edged graphene flakes, ${ }^{18}$ atomic reconstruction to zigzag edge by Joule heating, ${ }^{19}$ and electron beam irradiation ${ }^{20}$ ) implied the formation of the zigzag-type atomic structure of the pore edges by the reconstruction of the edge atomic structure. Moreover, observation of the ferromagnetism induced by decreasing the interpore distance and of the high density of the polarized spins at the pore edges by magnetic force microscope in the ferromagnetic graphene nanopore arrays (FGNPAs) ${ }^{13,14}$ suggested that the observed ferromagnetism was attributed to the presence of the polarized spins existing at the pore edges. Two theoretical analysis (GNR theory and Lieb's theorem ${ }^{21,22}$ ) of the observed magnetization values ( $\sim 0.3 \mu_{\mathrm{B}} /$ edge dangling bond) also suggested that the mono-hydrogenated zigzag pore edges were the origin for the ferromagnetism. ${ }^{13,15}$ Recent our work has proved 

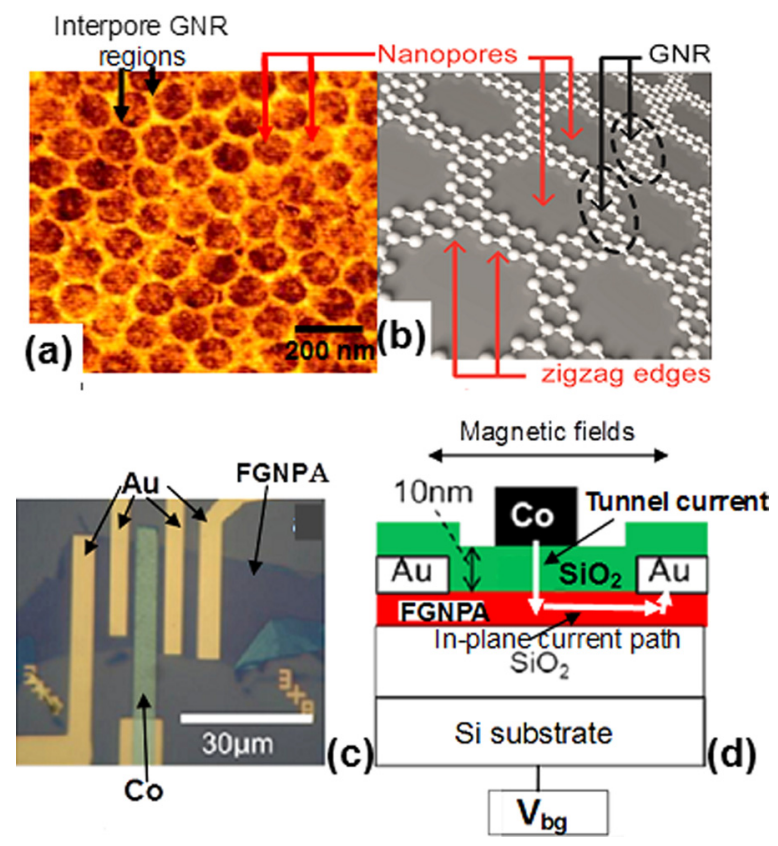

FIG. 1. (a) AFM image of the FGNPA, which consists of a honeycomb-like array of hexagonal nanopores. The typical interpore distance is $\sim 30 \mathrm{~nm}$ and the pore diameter is $\sim 80 \mathrm{~nm}$. (b) Schematic top view of a GNPA with zigzag-type atomic structure at the pore edges Interpore regions correspond to zigzag-type GNRs (e.g., with width of $\sim 30 \mathrm{~nm}$ ), which are onedimensional strip lines of graphenes. In actual samples, larger number of carbon unit cells exists in one interpore-GNR region. (c) Optical microscope image of a top view of the electrode pattern of the $\mathrm{Co} / \mathrm{SiO}_{2} / \mathrm{FGNPA}$ TMR junction. MR between the top Co electrode and the Au electrode located at the nearest right side was measured under constant current mode of $1 \mathrm{nA}$. (d) Schematic cross section of the TMR junction. The white line illustrates the constant current path. Thickness of the $\mathrm{SiO}_{2}$ tunnel barrier of $\sim 10 \mathrm{~nm}$ was confirmed by ellipsometry. Magnetic fields were applied in parallel with the FGNPA plane. A back gate voltage $\left(\mathrm{V}_{\mathrm{bg}}\right)$ was applied from the back side of the $\mathrm{Si}$ substrate via surface $\mathrm{SiO}_{2}$ film of $\mathrm{Si}$ substrate.

this by increasing saturation magnetization in mostly 100 times by increasing area of mono-hydrogen termination of the pore edges using specified resist treatment. ${ }^{15}$ The edge states were also confirmed by observation using ionic liquid gate. ${ }^{16}$ Also from other viewpoints, GNPAs are highly expected as quantum-information, ${ }^{23}$ optical, ${ }^{24}$ and semiconductingoperation $^{25}$ devices.

In the present study, TMR junctions utilizing such FGNPAs as ferromagnetic electrodes are realized. The fabricated TMR junction consists of $\mathrm{Co} / \mathrm{SiO}_{2} / \mathrm{FGNPA}$ (Figs. 1(c) and $1(\mathrm{~d})$ ), serving as a prototype structure for rare-metal free graphene-based TMR. The honeycomb-like array of hexagonal nanopores forming the FGNPA is shown in the atomic force microscope image (Fig. 1(a)) and in a schematic view (Fig. 1(b)). Fabrication followed our previous non-lithographic method, ${ }^{13-16}$ which realized the low defects and low contamination of the zigzag pore edges.

The fabricated TMR structure using this FGNPA as oneside electrode with the optical microscope is shown in Fig. 1(c) (top-view) and is schematically described in Fig. 1(d). The TMR behavior is measured along the constant current path illustrated in Fig. 1(d). The TMR ratio is defined as the difference between the resistance values $\left(R_{B}\right)$ at individual magnetic fields $(B)$ and the minimum MR value $\left(\mathrm{R}_{\min }\right)$, which is assumed to be the best parallel spin alignment between Co electrode and FGNPA; i.e., TMR ratio $=\left(R_{B}-R_{\text {min }}\right) / R_{\text {min }}$. A magnetization measurement of the overlayered structure of the $\mathrm{SiO}_{2} /$ FGNPA implies the persistence of the ferromagnetic signal even after evaporation of the $\mathrm{SiO}_{2}$ film on the pore edges, although the magnitude is reduced somewhat compared with that without $\mathrm{SiO}_{2}$ film.

Figures 2(a) and 2(b) give the result of a typical TMR measurement of the $\mathrm{Co} / \mathrm{SiO}_{2} / \mathrm{FGNPA}$ junction (shown in Figs. 1(c) and 1(d)) under in-plane parallel $B$ at (a) $1.5 \mathrm{~K}$ and (b) $300 \mathrm{~K}$. We identify the minimum resistance $\left(\mathrm{R}_{\min }\right)$ as the situation in which the external $B$ induces best matching between the spin polarizations of the magnetic materials (i.e., parallel spin alignment between cobalt and FGNPA) and take this as $R_{P}$ in subsequent calculations of TMR ratios, as mentioned in the figure caption. The observed TMR behaviors in Fig. 2(a) are significantly different from those of any other conventional TMR junctions. They exhibit the following unique $B$-dependent characteristics. (1) TMR ratio is tuned by the $B$ and can reach a signal about $20 \%$. (2) The minimum of TMR ratio appears as $B$ approaches to zero in $-B$ region. (3) The TMR ratios increases gradually crossing $B=0$. (4) The peak of TMR ratio emerges in $+B$ region. A similar behavior is observed for polarity changes in $B$, i.e., when sweeping from $+B$ to $-B$ (the black line in Fig. 2). As illustrated in the curves around zero-TMR ratio, the $\mathrm{Co} /$ $\mathrm{SiO}_{2}$ /bulk graphene junction (i.e., without the nanopores) shows drastically different behavior, with showing no clear TMR signatures. This suggests that the TMR-like behavior
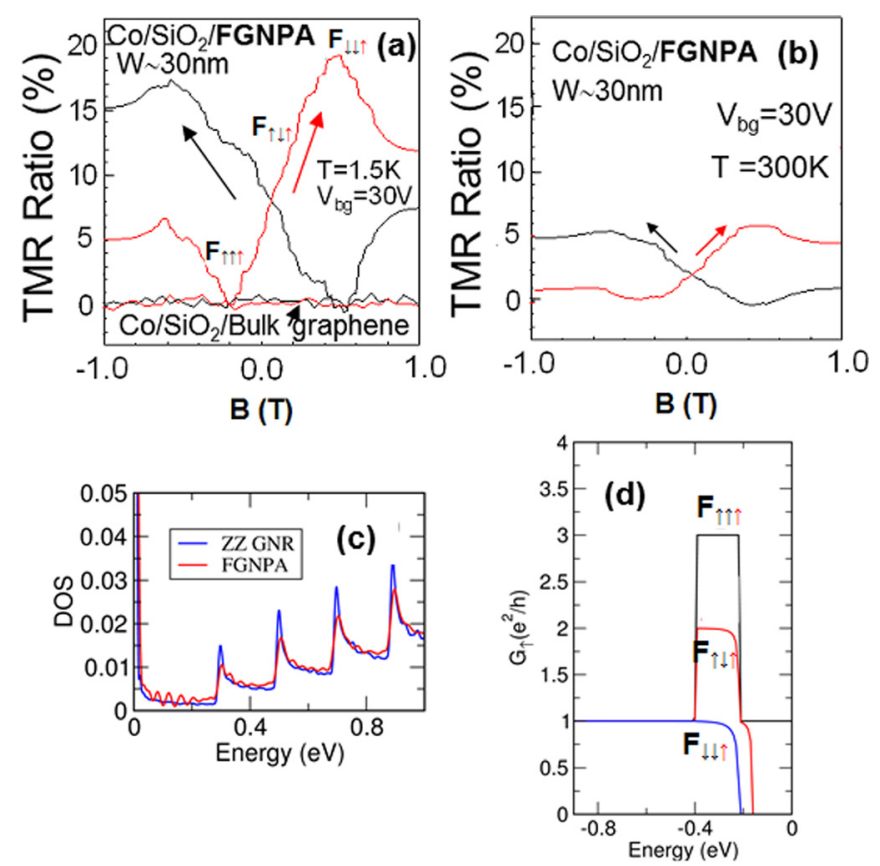

FIG. 2. The result of a typical TMR measurement of the $\mathrm{Co} / \mathrm{SiO}_{2} / \mathrm{FGNPA}$ junction (shown in Figs. 1(c) and 1(d)) under in-plane parallel $B$ at (a) $1.5 \mathrm{~K}$ and (b) $300 \mathrm{~K}$. In-plane magnetic field sweeps were performed from $B=-1$ to $+1 \mathrm{~T}$ (red line) and $B=+1$ to $-1 \mathrm{~T}$ (black line). Mostly constant zero TMR ratios shown in (a) are TMR values for the bulk graphene/ $\mathrm{SiO}_{2} / \mathrm{Co}$ junction. Notation $\mathrm{F}_{\uparrow \uparrow \uparrow}$ means spin configuration of the two edges of an interpore GNR region in the FGNPAs (left two black-arrows) and the Co (right one red-arrow). (c) Density of states calculated for the GNPA with an interpore distance of $W \sim 10 \mathrm{~nm}$ and a pore diameter of $\sim 80 \mathrm{~nm}$ and the related zigzag GNR. (d) Conductance between zigzag GNR junctions with different edge spin polarization states and cobalt electrode. Note the distinct differences in the MR signal in the energy regime where edge states dominate the electronic properties of the GNR. 
observed in the $\mathrm{Co} / \mathrm{SiO}_{2} / \mathrm{FGNPA}$ junction is unique to the present TMR structure, and is driven by spin tunneling properties between the Co electrode and the FGNPA. Present reproducibility of the TMR behaviors is over $80 \%$, because six of seven samples showed similar behaviors to date.

TMR properties observed at room temperature are shown in Fig. 2(b). Maximum TMR ratios decreases from $\sim 20 \%$ to $\sim 5 \%$ owing to the reduced flat-band ferromagnetism and the behaviors become much ambiguous compared with those at low temperatures, showing non-zero TMR ratios at high $B$ due to thermal spin instability. It should be noticed that, nevertheless, TMR behaviors are still observable.

The observed unique TMR behavior can be qualitatively understood by considering the spin alignment between two opposing pore edges of the FGNPA (i.e., two edges of the interpore GNR region in Fig. 1(b)) and the Co electrode. It also clarifies spin ground states of the H-terminated zigzagtype GNRs under no $B$. We support these interpretations by atomistic simulations. First, we calculate the density of states (DOS) of a GNPA reminiscent of the fabricated structure. The calculated DOS (Fig. 2(c)) is compared to that obtained for an infinitely long zigzag GNR (i.e., interpore GNR region in Fig. 1(b)). Ignoring the tunnel barrier, the TMR device is thus conveniently visualized as a junction between a Co spin injector and an array of uncoupled zigzag GNRs, the spin polarizations of which depend on the $B$. Based on this result, we simulate the spin transport properties of the device via a zigzag GNR-based junction, in which the spin polarization is differentiated between left (Co spin injector) and right (FGNPA) parts (Fig. 3(d)). We focus on the sweep from $-B$ to $+B$ (red curve in Fig. 2(a)).

As indicated in Fig. 2(d), we identify three distinct regions in the TMR response, corresponding to the situations where the magnetization of the FGNPA (i.e., spin alignment of two edges of the interpore GNRs) is ferromagnetic $\left(\mathrm{F}_{\uparrow \uparrow}\right.$ : the left

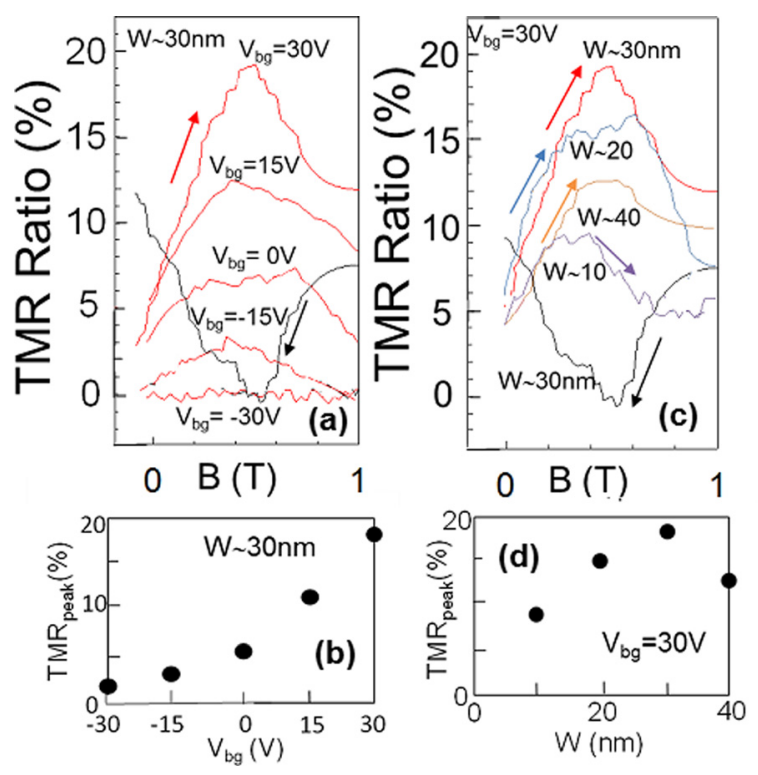

FIG. 3. (a) Measurements of the sample shown in Fig. 2 for $\mathrm{V}_{\mathrm{bg}}$ from $+30 \mathrm{~V}$ to $-30 \mathrm{~V}$ are shown by red curves, while a black line shows that for $-B$ to $+B$ regions for reference. (b) $\mathrm{V}_{\mathrm{bg}}$ dependence of peak values of the TMR ratios shown in Fig. 3(a). (c) Measurements of a sample similar to that in Fig. 2(a) but with different $W$. Exhibited $B$ regions are the same as Fig. 3(a). (d) $W$ dependence of peak values of the TMR ratios shown in Fig. 3(c). two black arrows at the subscript in the figure mean the spin moment of two edges of the interpore GNR) with spins parallel to the spin injector $(\mathrm{Co})\left(\mathrm{F}_{\uparrow \uparrow \uparrow}\right.$ : the right one red subscript arrow in the figure is the spin moment of injector), antiferromagnetic $\left(\mathrm{AF}, \mathrm{F}_{\uparrow \downarrow \uparrow}\right.$ ), and ferromagnetic with spins antiparallel to the spin injector $\left(\mathrm{F}_{\downarrow \downarrow \uparrow}\right)$. At $-B$, the spins of the injector and the FGNPA are largely parallel $\left(\mathrm{F}_{\uparrow \uparrow \uparrow}\right)$, resulting in maximum conductance through the junction (Fig. 2(d)) and thus the minimum of the TMR ratio in Fig. 2(a). As the $B$ approaches zero, the GNR array (i.e., ensemble of the two edges of the interpore GNRs) gradually transitions to an AF configuration $\left(\mathrm{F}_{\uparrow \downarrow}\right)$, where half of the conductance channels are suppressed, resulting in the half value of conductance (Fig. 2(d)) and gradual increase in the TMR ratio $\left(\mathrm{F}_{\uparrow \downarrow \uparrow}\right)$. As the $B$ is increased further to $+B$, the spin polarization of the GNR array changes to the $\left(\mathrm{F}_{\downarrow \downarrow}\right)$ configuration, suppressing all edge conductance channels, resulting in the conductance minimum (Fig. 2(d)) and subsequent the peak of TMR ratio observed around $B=0.5 \mathrm{~T}\left(\mathrm{~F}_{\downarrow \downarrow \uparrow}\right)$. These calculations suggest that gradual changes in TMR ratios can be actually possible. At higher $+B$, the spins of the Co injector start to align with the magnetic field, gradually quenching the TMR ratio, while non-zero TMR ratios in higher $+B$ regions suggest instability of the pore edge spins of the FGNPAs as well as those in $-B$ region.

It implies that the edge polarized spins of the interpore GNR regions can actually tunnel through $\mathrm{SiO}_{2}$ barrier forming the spin alignment with the spins of Co electrode and also that the spin ground states of the H-terminated zigzagGNRs are AF under no $B$. This result is consistent with our previous experimental results, which exhibited ferromagnetism only when $B$ is applied. ${ }^{13,14}$ It is important to note that the main mechanism revealed by our simulations does not depend on how the electrons are really injected from the cobalt electrode to the GNPA, but how efficiently spinpolarized electrons injected on the GNPA are further transmitted depending on the magnetic ordering of the local moments along the zigzag edges.

Here, we also demonstrate that the TMR characteristics can be controlled by changing a back gate voltage $\left(\mathrm{V}_{\mathrm{bg}}\right.$ : Fig. $\left.1(\mathrm{~d})\right)$ (Figs. 3(a) and 3(b)), as well as to the interpore spacing ( $W$, i.e., width of interpore GNRs in Fig. 1(b)) (Figs. 3(c) and $3(\mathrm{~d})$ ). The TMR ratio exhibits a maximum value of $\sim 20 \%$ at $\mathrm{V}_{\mathrm{bg}}=+30 \mathrm{~V}$ (Figs. 3(a) and 3(b)), while it is reduced with decreasing $\mathrm{V}_{\mathrm{bg}}$ toward $-\mathrm{V}_{\mathrm{bg}}$ region. This is consistent with the presence of evident spin-based phenomena observed only at $\mathrm{V}_{\mathrm{bg}}=+30 \mathrm{~V}$ for previous in-plane MR behaviors of FGNPAs. ${ }^{13}$ This is because the Au electrode was placed at the side position of the $\mathrm{Co} / \mathrm{SiO}_{2} / \mathrm{FGNPA}$ junction and the inplane spin current path exists through the H-terminated FGNPA between the TMR junction and the Au electrode in the present specified sample structure (Fig. 1(d)). Although the polarized spins at the pore edges and TMR ratio themselves have basically no correlation with the $\mathrm{V}_{\mathrm{g}}$, the spin current through the in-plane path is significantly reduced with decreasing $\mathrm{V}_{\mathrm{g}}$ due to the $\mathrm{n}$-type semiconducting behavior of the interpore GNR regions of the GNPA. ${ }^{13}$ This makes TMR features difficult to detect, resulting in the decreases in the observed TMR ratios.

For larger interpore spacing of $W \sim 40 \mathrm{~nm}$, the TMR value further decreases significantly (Figs. 3(c) and 3(d)). 

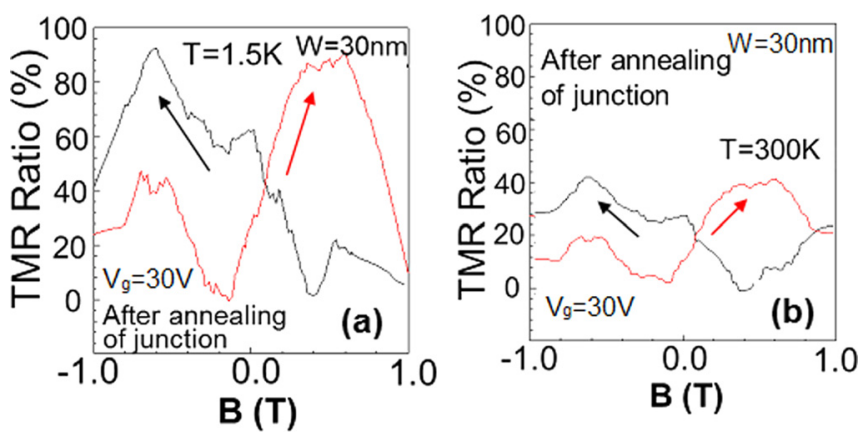

FIG. 4. TMR behaviors of the $\mathrm{Co} / \mathrm{SiO}_{2} / \mathrm{FGNPA}$ junction improved by annealing at (a) $1.5 \mathrm{~K}$ and (b) $300 \mathrm{~K}$. The annealing of $\mathrm{SiO}_{2} /$ FGNPA junction at $500^{\circ} \mathrm{C}$ was carried out under high vacuum atmosphere right after deposing of $\mathrm{SiO}_{2}$ layer on FGNPA.

For such a large spacing, the spin polarization of opposing pore edges (i.e., two edges of interpore GNRs) becomes unstable due to suppressed spin interaction, ${ }^{13}$ quenching the TMR properties. TMR ratios also decrease when using FGNPAs with smaller $W$ (Figs. 3(c) and 3(d)), because $W \leq 20 \mathrm{~nm}$ is too narrow to form spin currents along the in-plane current path through the FGNPA to $\mathrm{Au}$ electrode (Fig. 1(d)). Although ferromagnetism is stronger for smaller $W,{ }^{13}$ induced scattering by the nanopore array heavily obstructs spin flow to the Au electrode, reducing the TMR ratio. This is again consistent with previously observed spin-based phenomena in FGNPAs with $W \sim 30 \mathrm{~nm} .{ }^{13}$ Therefore, the optimum $W$ value exists for the TMR ratios.

We have presented unique TMR behaviors in the $\mathrm{Co} /$ $\mathrm{SiO}_{2} /$ FGNPA junctions. Nevertheless, the maximum TMR ratio is as low as $\sim 20 \%$ at best (Fig. 2(a)). One of the main reasons is the poor interaction at the lattice-mismatched $\mathrm{SiO}_{2} /$ FGNPA interface, particularly at the H-terminated pore edges, which destructs transport of the spin-alignment current and reduces TMR ratios. In contract, as shown in Fig. 4(a), we find that annealing of the $\mathrm{SiO}_{2} / \mathrm{FGNPA}$ structure at $\sim 500^{\circ} \mathrm{C}$ right after the deposition of $\mathrm{SiO}_{2}$ tunneling layer drastically (i.e., $\geq 5$ times) improves the TMR ratios of Fig. 2(a), although some instability of the pore edge spins still remain as some irregular small TMR ratio peaks. At room temperature, the maximum TMR ratio increases even by $\sim 10$ times (Fig. 4(b)) compared to that in Fig. 2(b).

This promises that introducing a lattice-matched tunnel barrier layer (e.g., graphene oxide) instead of $\mathrm{SiO}_{2}$ layer and also improvement of magnitude of the ferromagnetism in the FGNPAs (e.g., utilizing HSQ resist treatment ${ }^{15}$ ) can increase TMR ratios further and make evident room-temperature operation possible. Moreover, realization of FGNPA/(latticematched tunnel barrier)/FGNPA junction must significantly improve the performance of the TMR behaviors toward $\sim 1000 \%$ order.

The authors thank T. Nakamura, J. Kamijyo, K. Fujita, Y. Hashimoto, Y. Iye, S. Katsumoto, M. Yamamoto, S.
Tarucha, H. Hibino, T. Ando, T. Enoki, M. Koshino, J. Akimitsu, T. Muranaka, Y. Yagi, and M. S. Dresselhaus for their technical contribution, fruitful discussions, and encouragement. This work at Aoyama Gakuin was partly supported by a Grant-in-aid for Scientific Research (Basic research A: 24241046) in MEXT and AFOSR grant. The work by J.G.P. was financially supported by the Danish Council for Independent Research, FTP Grant Nos. 11105204 and 11-120941. S.R. and D.S. acknowledge financial support by the Spanish Ministry of Economy and Competitiveness (MAT2012-33911).

${ }^{1}$ M. N. Baibich, J. M. Bruto, and A. Fert, Phys. Rev. Lett. 61, 2472 (1988).

${ }^{2}$ J. S. Moodera, L. R. Kindler, T. M. Wong, and R. Meservey, Phys. Rev. Lett. 74, 3273-3276 (1995).

${ }^{3}$ Y. Yuasa, T. Nagahama, A. Fukushima, Y. Suzuki, and K. Ando, Nat. Mater. 3, 868-871 (2004).

${ }^{4}$ H. Munekata, H. Ohno, and S. von Molnár, Phys. Rev. Lett. 63, 1849-1852 (1989).

${ }^{5}$ H. Ohno, H. Munekata, T. Penney, S. von Molár, and L. L. Chang, Phys. Rev. Lett. 68, 2664-2667 (1992).

${ }^{6}$ P. N. Hai, S. Ohya, M. Tanaka, S. E. Barnes, and S. Maekawa, Nature 458, 489-492 (2009).

${ }^{7}$ K. Nakada, M. Fujita, G. Dresselhaus, and M. S. Dresselhaus, Phys. Rev. B 54, 17954-17961 (1996).

${ }^{8}$ D. Soriano, N. Leconte, P. Ordejón, J. C. Charlier, J. J. Palacios, and S. Roche, Phys. Rev. Lett. 107, 016602 (2011).

${ }^{9}$ H. X. Yang, A. Hallal, D. Terrade, X. Waintal, S. Roche, and M. Chshiev, Phys. Rev. Lett. 110, 046603 (2013).

${ }^{10}$ T. Shimizu, J. Haruyama, D. C. Marcano, D. V. Kosinkin, J. M. Tour, K. Hirose, and K. Suenaga, Nat. Nanotechnol. 6, 45-50 (2011).

${ }^{11}$ J. Balakrishnan, G. K. W. Koon, M. Jaiswal, A. H. C. Neto, and B. Özyilmaz, Nat. Phys. 9, 284-287 (2013).

${ }^{12}$ J. Kamijyo, T. Nakamura, T. Kato, and J. Haruyama, "Spin-orbit-interaction induced electron spin coherence in slightly hydrogenated graphene within a topological insulating regime," Science (unpublished), available at http:// www.ee.aoyama.ac.jp/haru-lab/.

${ }^{13}$ K. Tada, T. Hashimoto, J. Haruyama, H. Yang, and M. Chshiev, Phys. Status Solidi B 249, 2491-2496 (2012).

${ }^{14}$ T. Shimizu, J. Nakamura, K. Tada, Y. Yagi, and J. Haruyama, Appl. Phys. Lett. 100, 023104 (2012).

${ }^{15}$ T. Kato, T. Nakamura, J. Kamijyo, T. Kobayashi, Y. Yagi, and J. Haruyama, Appl. Phys. Lett. 104, 252410 (2014).

${ }^{16}$ T. Hashimoto, S. Kamikawa, Y. Yagi, and J. Haruyama, Mater. Sci. Appl. 5(1), 1-9 (2014).

${ }^{17}$ B. Krauss, P. N. Incze, V. Skakalova, L. P. Biro, K. von Klitzing, and J. H. Smet, Nano Lett. 10, 4544 (2010).

${ }^{18}$ Y. You, Z. Ni, T. Yu, and Z. Shen, Appl. Phys. Lett. 93, 163112 (2008).

${ }^{19}$ X. Jia, M. Hofmann, V. Meunier, B. G. Sumpter, J. Campos-Delgado, J. M. Romo-Herrera, H. Son, Y.-P. Hsieh, A. Reina, M. S. Dresselhaus et al., Science 323, 1701 (2009).

${ }^{20}$ Ç. Ö. Girit, J. C. Meyer, R. Erni, M. D. Rossell, L. Yang, C.-H. Park, M. F. Crommie, M. L. Cohen, S. G. Louie, A. Zettl et al., Science 323, 1705 (2009).

${ }^{21}$ E. H. Lieb, Phys. Rev. Lett. 62, 1201-1204 (1989).

${ }^{22}$ M. L. Trolle, U. S. Møller, and T. G. Pedersen, Phys. Rev. B 88, 195418 (2013).

${ }^{23}$ T. G. Pedersen, C. Flindt, J. Pedersen, N. A. Mortensen, A. P. Jauho, and K. Pedersen, Phys. Rev. Lett. 100, 136804 (2008).

${ }^{24}$ T. G. Pedersen, C. Flindt, J. Pedersen, A. P. Jauho, N. A. Mortensen, and K. Pedersen, Phys. Rev. B 77, 245431 (2008).

${ }^{25}$ J. A. Fürst, J. G. Pedersen, C. Flindt, N. A. Mortensen, M. Brandbyge, T. G. Pedersen, and A.-P. Jauho, New J. Phys. 11, 095020 (2009). 